in vivo $35: 2889-2894(2021)$

doi:10.21873/invivo.12578

\title{
Modulation of Immune System - Strategy in the Treatment of Breast Cancer
}

\author{
DUMITRU CRISTINEL BADIU ${ }^{1}$, ANCA ZGURA ${ }^{2}$, LAURENTIA GALES ${ }^{2}$, \\ LAURA ILIESCU ${ }^{3}$, RODICA ANGHEL ${ }^{2}$ and BOGDAN HAINEALA ${ }^{4}$ \\ ${ }^{1}$ General Surgery Department, "Bagdasar Arseni” Clinical Emergency Hospital, \\ "Carol Davila” University of Medicine and Pharmacy, Bucharest, Romania; \\ ${ }^{2}$ Department of Oncology-Radiotherapy, Prof. Dr. Alexandru Trestioreanu Institute of Oncology, \\ Carol Davila University of Medicine and Pharmacy, Bucharest, Romania; \\ ${ }^{3}$ Department of Internal Medicine II, Fundeni Clinical Institute, \\ Carol Davila University of Medicine and Pharmacy, Bucharest, Romania; \\ ${ }^{4}$ Department of Urology, "Fundeni" Clinical Institute, \\ "Carol Davila” University of Medicine and Pharmacy, Bucharest, Romania
}

\begin{abstract}
Background/Aim: In women, breast cancer is the most commonly diagnosed cancer type and at the same time the main cause of cancer-related death. Many mechanisms are involved in the tumor microenvironment to restrict the anti-tumor activity by the immune system. Identification of novel prognostic tools based on immunological data could make significant impact in developing innovative immunotherapy strategies that will restore the anti-tumor immune system efficacy. Patients and Methods: The study was performed on patients diagnosed with breast cancer, who were divided into two groups depending on the expression of HER2. For the studied group, first we described the infiltrate inflammatory on slides stained with haematoxylin eosin (HE) and in the second part we used flow cytometry in order to measure the percentage of $T$ lymphocytes from the peripheral blood before and after breast cancer treatment. Results: High presence of tumorinfiltrating lymphocytes (TILs) was associated with prognostic improvement, better disease-free survival, distant disease-free survival and overall survival. In breast cancer,
\end{abstract}

This article is freely accessible online.

Correspondence to: Anca Zgura, Department of OncologyRadiotherapy, Prof. Dr. Alexandru Trestioreanu Institute of Oncology, Carol Davila University of Medicine and Pharmacy, 37 Dionisie Lupu Street, Sector 2, 020021 Bucharest, Romania. Tel: +40 751110005, e-mail: medicanca@gmail.com

Key Words: Breast cancer, immune cells, lymphocytes, chemotherapy, radiotherapy. the presence of TILs predicts the full pathological response rate ( $p C R)$ after neoadjuvant chemotherapy. TILs are one of the best examples of the strict relationship existing between natural defence and carcinogenesis. Conclusion: Modulation of the immune system is a promising strategy in the treatment of breast cancer, especially in triple-negative and HER2positive molecular subtypes, the most immunogenic subtypes with a poor prognosis.

In women, breast cancer is the most commonly diagnosed cancer type, at the same time being the main cause of cancerrelated death (1). Many mechanisms are involved in the tumor microenvironment to restrict the anti-tumor activity by the immune system. Identification of novel prognostic tools based on immunological data could make significant impact in developing innovative immunotherapy strategies that will restore the anti-tumor immune system efficacy (2). The immune cells functionally regulate the development and progression of breast cancer. Tumour progression has 3 phases: elimination, balance, and escape. In the elimination and balance phases, the cancer cells are predominantly attacked by CD $8+\mathrm{T}$ lymphocytes, in the escape phase the tumour inhibits CD8 + T lymphocytes, this is the arsenal of the immune system (3). The specific markers for the T, B and NK cells are CD3, CD19, respectively CD16+56. T lymphocytes play a vital function in constraining tumor development and in breast cancer CD4+ and CD8+ infiltrating $\mathrm{T}$ lymphocytes, are abundant in hight-grade ductal carcinoma in situ as well as invasive carcinomas (46). B lymphocytes participate at the humoral specific immune response. B CD19+ lymphocytes inhibit the activity of helper $\mathrm{T}$ lymphocytes, indirectly inhibiting the 
differentiation of $\mathrm{B}$ cells. They negatively regulate the humoral and cellular immune response of cellular T subsets. NK cells can serve as the first line of defence in association with CD8+ T lymphocytes (7). NK cells infiltrate breast lesions in a proportion of approximately $5 \%$ of the total lymphocyte population (8). Recently, it has been observed that low levels of NK cells may be apparently linked to more unfavourable clinical outcomes. The role of cytotoxic $\mathrm{T}$ lymphocytes is to destroy infected target cells. Suppressor T lymphocytes represent a subpopulation of $\mathrm{T}$ lymphocytes, the existence of which is controversial. Those lymphocytes have inhibitor effects in the immune response.

Tumor-infiltrating lymphocytes (TILs) were initially considered a reaction of the host to the tumour, later they were proven to provide prognostic and potentially predictive value. Now inflammatory infiltrate is framed in the morphofunctional notion of tumour microenvironment (along with the other elements of the tumour stroma, fibroblasts, myofibroblasts, cells endothelial, extracellular matrix) complex system providing various and important cytokine signals supporting neoplastic progression and invasion (9).

In practice, it was noticed that patients with breast neoplasms included in the same prognostic group, (lymph node status, histological subtype, presence or absence of hormonal receptors, KI67 index value, HER2-positive or negative tumors) who followed the same treatment, have different disease evolution, and this leads to the idea that there is a series of other factors affecting this evolution and the immune system plays an important role (10).

The decrease of the immune status of patients with cancer has an impact on the numerical balance of lymphoid cells from the peripheral circulation and this is showing up a deviation from the normal percentual values of total $\mathrm{T}$ lymphocytes. Therefore, establishing the CD3+, CD4+ and CD8+ cell proportions is useful in monitoring patients with immune status affected by the cancer and, and it is possible to correlate the values of obtained parameters with response to treatments like chemotherapy, immunotherapy or radiotherapy (11).

\section{Patients and Methods}

The study was performed on patients diagnosed with breast cancer and were divided into two groups depending on the overexpression of HER2. For the studied group, we first described the infiltrate inflammatory on standard slides stained with haematoxylin eosin (HE) (Figure 1) and in the second part we used the principle of flow cytometry in order to measure the percentage of T lymphocytes in the blood.

The quantification of the infiltrate was performed after a thorough analysis of each case in part, and a score was designed in 3 degrees according to the amplitude of the inflammatory cellularity. Determining the percentages of CD4 and CD8 T lymphocytes is useful in assessing the immune status of the patient undergoing specific cancer treatment.

\section{Results}

From the total number of patients included in the study, $39.3 \%$ showed HER 2 overexpression. From the analysis of the TILs in relation with overexpression of HER2, 11 patients presented low TILs, 8 moderate and 3 high TILs, and in the group of patients with HER-2/neu-negative, 14 had low TILs, 13 moderate and 6 patients had high TILs.

From the analysis of patients with positive lymph nodes, it was observed that they were associated with TILs represented in a low and medium percentage. The analysis showed a significant correlation between TILs and lymph status. The patients with negative lymph nodes had rich lymphocytes infiltrate.

In this study, we found that HER-2/neu-positive tumours and triple-negative subtype (TNBCs) showed low TILs, suggesting that decreased immune response plays an important role in disease progression.

Determination of the dynamics of lymphocyte populations. The therapeutic effect of trastuzumab depends on acquired and adaptive immune-mediated mechanisms, and this effect has been shown in recent studies. Studies confirmed that disease-free survival and overall survival are influenced by high levels of TILs in HER2-enriched by using therapeutic effects of anti-HER2 antibody with a combination therapy that inhibits the PI3K/AKT pathway (12).

Interestingly, for patients diagnosed with breast cancer in the metastatic stage undergoing oral chemotherapy with capecitabine, $\mathrm{T}$ cell percentage was associated with smaller changes in the proportions of CD8+ and CD4+ between the first series of chemotherapy and after the second series of treatment.

There are contradictory data in the literature on the number of CD4 $\mathrm{T}$ lymphocytes in the blood and migrating to the intra-tumoral or peritumoral area at the time of disease progression.

There are studies showing that tumour regression is associated with a low number of CD4+ T lymphocytes and studies showing that an increased percentage of CD4+ T lymphocytes is associated with the response to treatment (1315).

In the case of the patient undergoing radiotherapy, an increase in CD4+ T lymphocyte levels is observed, followed by a decrease one month after radiotherapy (Figure 2). In the case of a patient undergoing treatment with trastuzumab and hormonal treatment, a decrease is initially observed followed by an increase in CD4+ $\mathrm{T}$ values. The patient undergoing treatment with docetaxel and trastuzumab initially maintained a plateau phase between the 2 assessments followed by a decrease at the last assessment. For the patient who received neoadjuvant treatment, there was a decrease in evaluation after the first series of chemotherapy followed by an increase in values after the second series. 


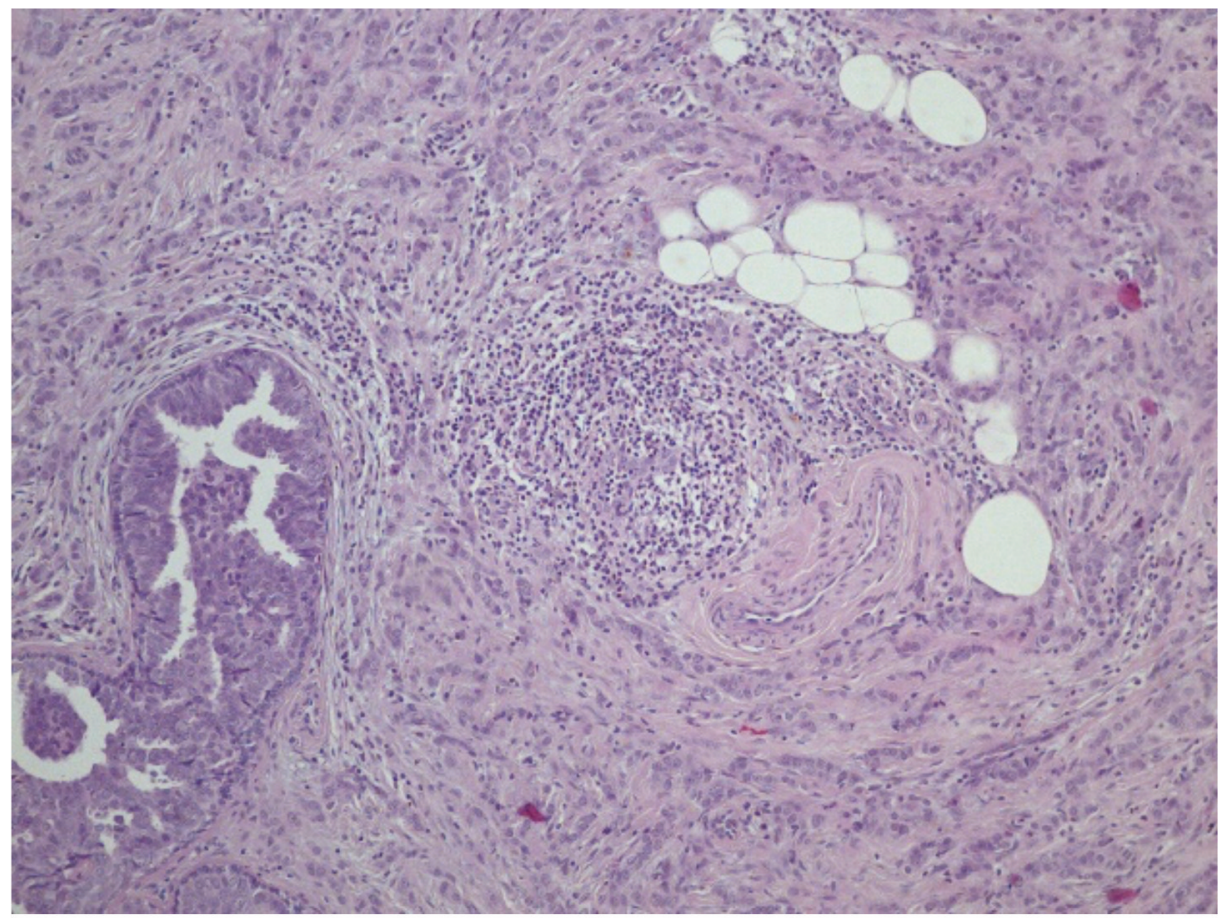

Figure 1. Histopathological evaluation of tumour lymphocyte infiltrate.

In the case of the patient undergoing radiotherapy, it is observed that similar values of CD8+ T lymphocytes were maintained (Figure 3). In the case of the patient undergoing treatment with Trastuzumab and hormonal treatment, a decrease $(8.85 \%)$ is initially observed, followed by an increase in values (16.25\%). The patient undergoing treatment with docetaxel and trastuzumab initially observed an increase in values $(21.6 \%)$, followed by a decrease $(13.58 \%)$. In the patient who received neoadjuvant treatment, a decrease is observed after the first series (11.11\%) followed by an increase after the second series $(29.22 \%)$ of treatment.

\section{Discussion}

During the past decade, insight has been gained regarding mechanisms underlying the dynamic interplay between immune cells and tumor progression. The accumulated data indicate that the outcome of an immune response toward a tumor is largely determined by the type of immune response. A number of studies showed that, in patients with cancer the number of CD4+ and CD8+ T lymphocytes are low at the time of diagnosis. But the assessment of lymphocyte subsets by using monoclonal antibodies in those patients offer different results, partially depending on the type of tumour, disease stage and body defence system.

The lymphocyte infiltrate in breast cancer was widely researched by now; however, even if it is usually accepted, the fact that high presence of TILs is associated with the prognostic improvement, the correlation between different TILs subpopulations, and the responses experimentally observed is still marked by a certain level of controversiality (11). It is considered that $75 \%$ from the TILs are T cells.

Loi et al. in 2013 were the first to document a correlation between higher levels of TILs present at diagnosis with decreased distant recurrence rates in triple-negative breast cancer patients. Subsequently, this hypothesis was supported by numerous authors, who evaluated it in current and future clinical trials and in diagnosis (15-17).

The study performed on a total number of 2009 samples from the clinical study BIG 02-98 adjuvant stage III, highlighted a linear correlation between high quantities of TILs and increased risk of relapse and death, notwithstanding the type of chemotherapy scheme (anthracycline $v s$. doxorubicin and docetaxel). Among the population with positive HER2 breast cancer, the TILs were also associated with improved results in case of anthracycline-based treatment (18).

Case studies have correlated high TILs levels with a better response to preoperative neoadjuvant therapy and those with initial intervention. Neoadjuvant chemotherapy induces a stimulation of inflammatory stromal infiltration $(19,20)$.

The presence of axillary lymphatic nodes overshadows the prognosis. Examination of the lymph nodes has a role in the management of patients diagnosed with breast cancer (21). The presence of node metastasis is not only a diagnostic 


\section{Dynamics of CD4}

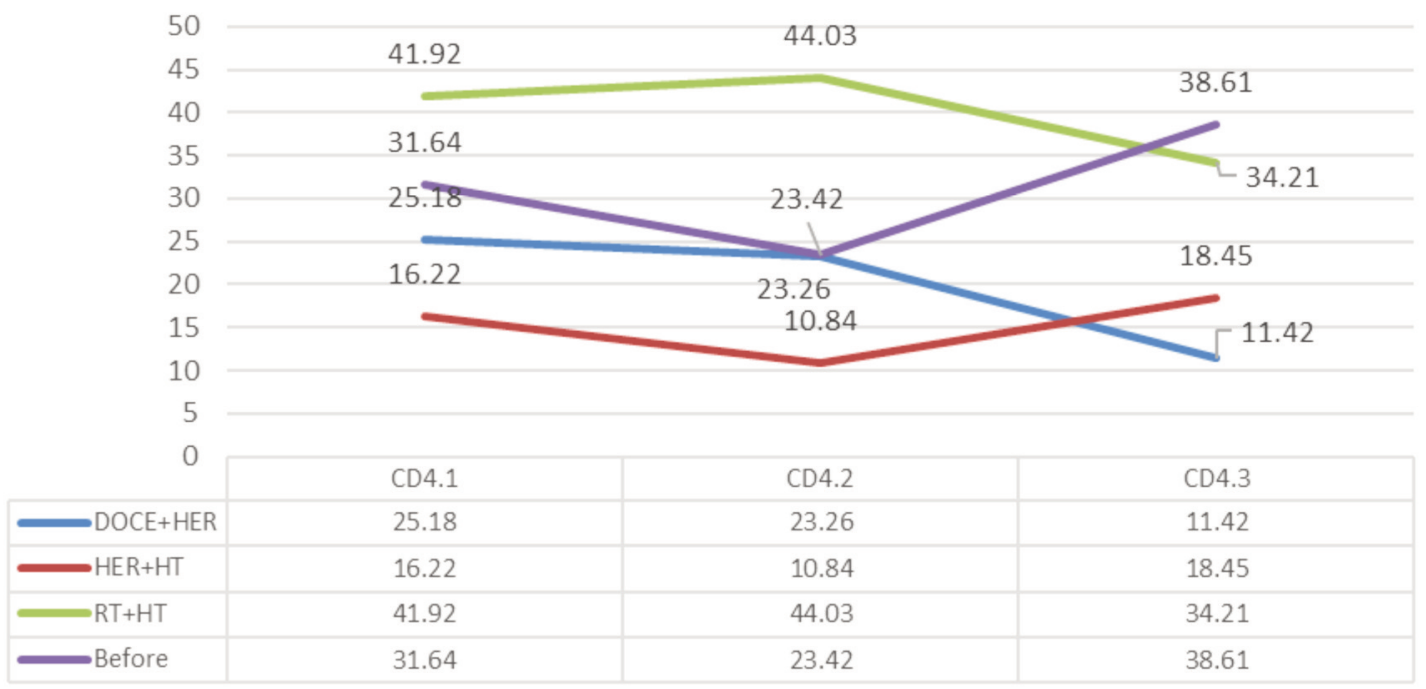

Figure 2. Evaluation of CD4+ Tlymphocytes depending on treatment. Doce $+H E R$ : Docetaxel + herceptine; HER $+H T:$ herceptine + hormonaltherapy; $R T+H T$ : radiotherapy + hormonal therapy.

\section{Dynamics of CD8}

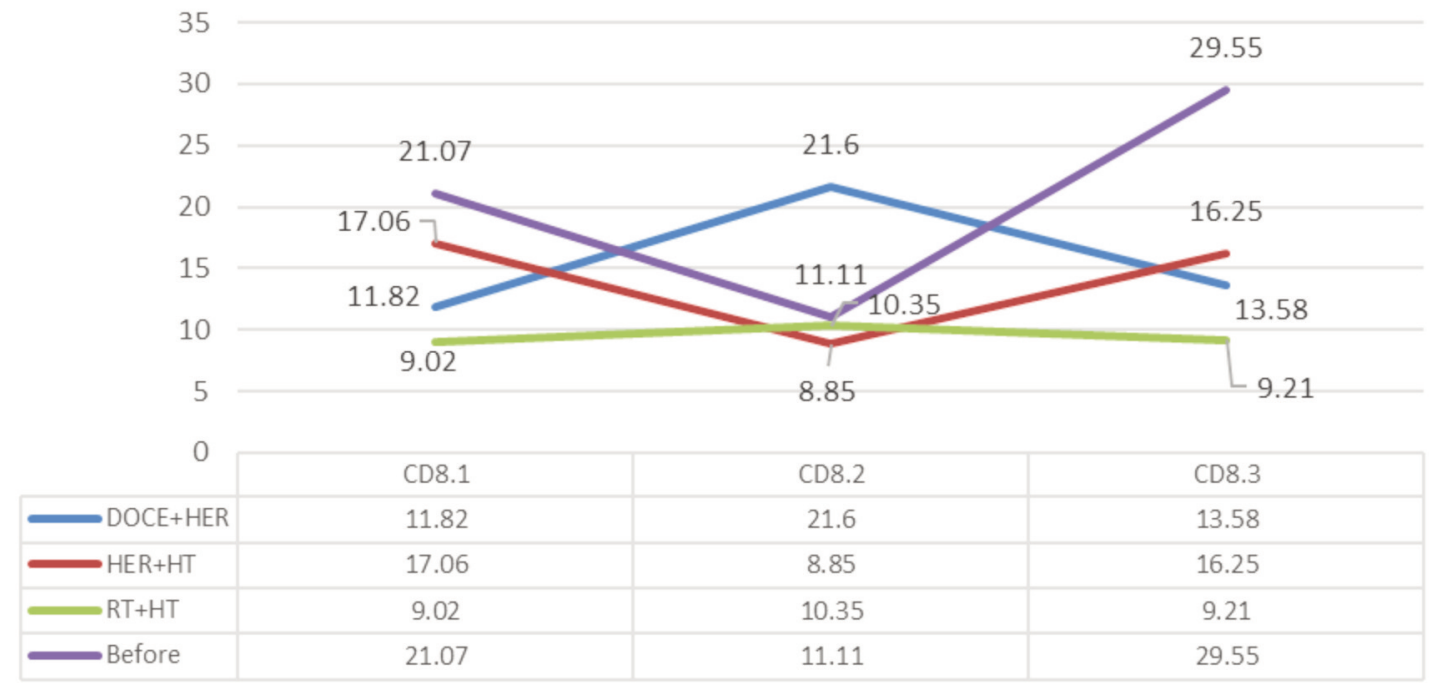

Figure 3. Evaluation of CD8+ T lymphocytes depending on treatment. Doce +HER: docetaxel + herceptine; HER+HT: herceptine+hormonal therapy; $R T+H T$ : radiotherapy + hormonal therapy.

marker, but also a marker of the presence of an aggressive phenotype. In this study, we noticed an association between the TILs presence in small percentages and positive nodes.

Analysis of lymphocytic tumour infiltrate related to patients' age demonstrated that the percentage of $\mathrm{T}$ lymphocytes was directly associated with the patient's age. Such a relationship was investigated in other studies with discordant results $(22,23)$ ascertaining that only $\mathrm{T}$ cells were directly associated to the patient's age partially supporting this way. 


\section{Conclusion}

Modulation of the immune system is a promising strategy in the treatment of breast cancer, especially in triple-negative and HER2-enriched tumors when considering intrinsic subtypes of breast cancer. The Nobel Prize for Physiology and Medicine 2018 was awarded to researchers James P. Allison (USA) and Tasuku Honjo (Japan) for discovering a therapy against cancer by inhibiting the negative regulation of the immune system.

The results of this study show that the evolution of neoplasia follows a complex path that paradoxically gathers discordant types of relationships between the immune system and the rest of the body, and TILS are one of the best examples and should become a test usually performed, especially concerning the most aggressive subtypes of breast cancer. Development of such a prognostic tool based on immunological data could make significant impact on the treatment and overall survival of cancer patients.

\section{Conflicts of Interest}

The Authors declare that they have no competing interests in relation to this study.

\section{Authors' Contributions}

$\mathrm{BH}$ was responsible for writing the manuscript. $\mathrm{AZ}, \mathrm{BH}$, and $\mathrm{DCB}$ were responsible for reviewing and editing the manuscript. AZ, LG and LI made substantial contributions to the conception or design of the work. RA were responsible for critically reviewing the manuscript. All Authors read and approved the final manuscript.

\section{References}

1 GLOBOCAN. Estimated Incidence, Mortality and Prevalence Worldwide in 2018. Available at: http://globocan.iarc.fr/Pages/ fact_sheets_cancer.aspx [Last accessed on July 15, 2021]

2 Bacinschi XE, Zgura A, Safta I and Anghel R: Biomolecular factors represented by Bcl-2, p53, and tumor-infiltrating lymphocytes predict response for adjuvant anthracycline chemotherapy in patients with early triple-negative breast cancer. Cancer Manag Res 12: 11965-11971, 2020. PMID: 33244272. DOI: $10.2147 / C M A R . S 274104$

3 Liu HZ, Deng W, Li JL, Tang YM, Zhang LT, Cui Y and Liang XQ: Peripheral blood lymphocyte subset levels differ in patients with hepatocellular carcinoma. Oncotarget 7(47): 77558-77564, 2016. PMID: 27813499. DOI: 10.18632/oncotarget.13041

4 Koretzky GA: Multiple roles of CD4 and CD8 in T cell activation. J Immunol 185(5): 2643-2644, 2010. PMID: 20724729. DOI: 10.4049/jimmunol.1090076

5 Baxevanis CN, Fortis SP and Perez SA: The balance between breast cancer and the immune system: Challenges for prognosis and clinical benefit from immunotherapies. Semin Cancer Biol 72: 7689, 2021. PMID: 31881337. DOI: 10.1016/j.semcancer.2019.12.018

6 Vazquez MI, Catalan-Dibene J and Zlotnik A: B cells responses and cytokine production are regulated by their immune microenvironment. Cytokine 74(2): 318-326, 2015. PMID: 25742773. DOI: $10.1016 /$ j.cyto.2015.02.007

7 Mahmoud SM, Paish EC, Powe DG, Macmillan RD, Grainge MJ, Lee AH, Ellis IO and Green AR: Tumor-infiltrating CD8+ lymphocytes predict clinical outcome in breast cancer. J Clin Oncol 29(15): 1949-1955, 2011. PMID: 21483002. DOI: 10.1200/JCO.2010.30.5037

8 Ruffell B, Au A, Rugo HS, Esserman LJ, Hwang ES and Coussens LM: Leukocyte composition of human breast cancer. Proc Natl Acad Sci USA 109(8): 2796-2801, 2012. PMID: 21825174. DOI: $10.1073 /$ pnas.1104303108

9 Liu F, Lang R, Zhao J, Zhang X, Pringle GA, Fan Y, Yin D, Gu $\mathrm{F}$, Yao $\mathrm{Z}$ and $\mathrm{Fu} \mathrm{L}$ : CD8+ cytotoxic $\mathrm{T}$ cell and FOXP3+ regulatory $\mathrm{T}$ cell infiltration in relation to breast cancer survival and molecular subtypes. Breast Cancer Res Treat 130(2): 645655, 2011. PMID: 21717105. DOI: 10.1007/s10549-011-1647-3

10 Ma Y, Mattarollo SR, Adjemian S, Yang H, Aymeric L, Hannani D, Portela Catani JP, Duret H, Teng MW, Kepp O, Wang Y, Sistigu A, Schultze JL, Stoll G, Galluzzi L, Zitvogel L, Smyth MJ and Kroemer G: CCL2/CCR2-dependent recruitment of functional antigen-presenting cells into tumors upon chemotherapy. Cancer Res 74(2): 436-445, 2014. PMID: 24302580. DOI: 10.1158/0008-5472.CAN-13-1265

11 Mahmoud S, Lee A, Ellis I and Green A: CD8(+) T lymphocytes infiltrating breast cancer: A promising new prognostic marker? Oncoimmunology 1(3): 364-365, 2012. PMID: 22737616. DOI: 10.4161/onci.18614

12 Wang W, Green M, Rebecca liu J, Lawrence T and Zou W: CD8+ $\mathrm{T}$ cells in immunotherapy, radiotherapy, and chemotherapy. Oncoimmunology: 23-39, 2019. DOI: 10.1007/978-3-319-62431$0 \_3$

13 Tao CJ, Chen YY, Jiang F, Feng XL, Jin QF, Jin T, Piao YF and Chen XZ: A prognostic model combining CD4/CD8 ratio and $\mathrm{N}$ stage predicts the risk of distant metastasis for patients with nasopharyngeal carcinoma treated by intensity modulated radiotherapy. Oncotarget 7(29): 46653-46661, 2016. PMID: 27270307. DOI: 10.18632 /oncotarget.9695

14 Loi S, Sirtaine N, Piette F, Salgado R, Viale G, Van Eenoo F, Rouas G, Francis P, Crown JP, Hitre E, de Azambuja E, Quinaux E, Di Leo A, Michiels S, Piccart MJ and Sotiriou C: Prognostic and predictive value of tumor-infiltrating lymphocytes in a phase III randomized adjuvant breast cancer trial in node-positive breast cancer comparing the addition of docetaxel to doxorubicin with doxorubicin-based chemotherapy: BIG 02-98. J Clin Oncol 31(7): 860-867, 2013. PMID: 23341518. DOI: 10.1200/JCO.2011. 41.0902

15 Dieci MV, Miglietta F and Guarneri V: Immune infiltrates in breast cancer: recent updates and clinical implications. Cells 10(2): 223, 2021. PMID: 33498711. DOI: 10.3390/cells 10020223

16 Zgura A, Gales L, Bratila E, Mehedintu C, Haineala B, Barac R, Popa A, Buhas C, Berceanu C, Andreescu C and Anghel R: Variation of the $\mathrm{T}$ lymphocytes according to treatment in breast cancer. Revista de Chimie 70(5): 1649-1654, 2020. DOI: 10.37358/RC.19.5.7186

17 Losurdo A, De Sanctis R, Fernandes B, Torrisi R, Masci G, Agostinetto E, Gatzemeier W, Errico V, Testori A, Tinterri C, Roncalli M and Santoro A: Insights for the application of TILs and $\mathrm{AR}$ in the treatment of TNBC in routine clinical practice. Sci Rep 10(1): 20100, 2020. PMID: 33208857. DOI: 10.1038/s41598-02077043-9 
18 Loi S, Michiels S, Salgado R, Sirtaine N, Jose V, Fumagalli D, Kellokumpu-Lehtinen PL, Bono P, Kataja V, Desmedt C, Piccart MJ, Loibl S, Denkert C, Smyth MJ, Joensuu H and Sotiriou C: Tumor infiltrating lymphocytes are prognostic in triple negative breast cancer and predictive for trastuzumab benefit in early breast cancer: results from the FinHER trial. Ann Oncol 25(8): 15441550, 2014. PMID: 24608200. DOI: 10.1093/annonc/mdu112

19 Jatoi I, Hilsenbeck SG, Clark GM and Osborne CK: Significance of axillary lymph node metastasis in primary breast cancer. J Clin Oncol 17(8): 2334-2340, 1999. PMID: 10561295. DOI: 10.1200/JCO.1999.17.8.2334

20 Lee H, Lee M, Seo JH, Gong G and Lee HJ: Changes in tumorinfiltrating lymphocytes after neoadjuvant chemotherapy and clinical significance in triple negative breast cancer. Anticancer Res 40(4): 1883-1890, 2020. PMID: 32234876. DOI: 10.21873/anticanres.14142

21 Marsigliante S, Biscozzo L, Marra A, Nicolardi G, Leo G, Lobreglio GB and Storelli C: Computerised counting of tumour infiltrating lymphocytes in 90 breast cancer specimens. Cancer Lett 139(1): 33-41, 1999. PMID: 10408906. DOI: 10.1016/ s0304-3835(98)00379-6
22 Vatner RE, Cooper BT, Vanpouille-Box C, Demaria S and Formenti SC: Combinations of immunotherapy and radiation in cancer therapy. Front Oncol 4: 325, 2014. PMID: 25506582. DOI: 10.3389 /fonc. 2014.00325

23 Yu H, Meng X, Chen H, Han X, Fan J, Gao W, Du L, Chen Y, Wang Y, Liu X, Zhang L, Ma G and Yang J: Correlation between mammographic radiomics features and the level of tumorinfiltrating lymphocytes in patients with triple-negative breast cancer. Front Oncol 10: 412, 2020. PMID: 32351879. DOI: $10.3389 /$ fonc. 2020.00412
Received May 23, 2021

Revised June 24, 2021

Accepted July 16, 2021 